\title{
Emerging Resistance in Neisseria gonorrhoeae at an STD Referral Centre
}

\author{
Syeda Summaiyah* and G. Jyothi Lakshmi \\ Department of Microbiology, Osmania Medical College, India \\ *Corresponding author
}

\begin{tabular}{l} 
Ke y w o r d s \\
$\begin{array}{l}\text { Neisseria } \\
\text { gonorrhoeae, STD, } \\
\text { Gonorrhoea, } \\
\text { Ciprofloxacin }\end{array}$ \\
\hline Article Info \\
$\begin{array}{l}\text { Accepted: } \\
\text { 05 February } 2020 \\
\text { Available Online: } \\
\text { 10 March } 2020\end{array}$ \\
\hline
\end{tabular}

\begin{abstract}
A B S T R A C T
Gonorrhoea is a sexually transmitted infection caused by Neisseria gonorrhoeae and is a major public health problem. Resistance has rapidly emerged to recommended antimicrobial agents for the management of gonorrhoea. This study was undertaken to determine the resistance of Neisseria gonorrhoeae among the clinically suspected cases of gonorrhoea in an STD Referral Centre. Urethral discharge and endocervical swabs were collected from clinically suspected cases of gonorrhoea. Gram's stain was performed to observe intracellular gram-negative diplococci. Blood agar and Chocolate agar were used for the growth of the organism. Antibiotic sensitivity testing was performed by the Kirby Bauer technique using the Calibrated Dichotomous Sensitivity method and CLSI method. Chromogenic cephalosporin test was done to identify Penicillinase Producing Neisseria gonorrhoea. Results: 3213 patients attended the STD Out Patient Department during the study period. From clinically suspected cases of gonorrhoea, 66 urethral discharge and 2 endocervical samples were collected. 17 (25\%) samples were positive for Neisseria gonorrhoea out of which eight were Penicillinase Producing Neisseria gonorrhoea. Neisseria gonorrhoeae was resistant to penicillin, ciprofloxacin and nalidixic acid. The results of our study demonstrated a sharp increase in the percentage of PPNG in India over the past two decades. Hence, studying the resistance patterns of gonorrhoea and identification of PPNG strains will greatly help with prescribing the most appropriate antimicrobials for the effective treatment of gonorrhoea.
\end{abstract}

\section{Introduction}

Gonorrhoea is a major public health concern globally causing substantial morbidity and economic burden. Although infections are symptomatic in males, they are asymptomatic in females leading to complications like PID, ectopic pregnancy and infertility among women ${ }^{(2)}$ Over the last decade, Neisseria gonorrhoeae has developed resistance against antimicrobial agents such as penicillin, azithromycin, tetracycline and quinolones in several countries including India. ${ }^{(2)}$
During 2009-12, the percentage of penicillinase- producing $N$. gonorrhoeae (PPNG), isolates from WHO South East Asian Region (SEAR) varied from 0\% (Pune, Nagpur) to $88.9 \%$ (Bhutan), while the rates of resistance to tetracycline and resistance to ciprofloxacin varied from 9.8\%-100\% and $57 \%-100 \%$, respectively ${ }^{(2)}$ The percentage of ciprofloxacin-resistant strains have varied in India in different parts of the country ${ }^{(2)}$

Two types of $N$. gonorrhoeae resistance to penicillin and tetracycline are identified. The 
first is chromosomally mediated acquisition of mutated genes or loci and the second is plasmid-mediated resistance due to the production of penicillinase. (3) Three main resistance plasmids (R-plasmids) are associated with the worldwide emergence of penicillinase-producing $N$. gonorrhoeae (PPNG). ${ }^{(3)}$ Due to paucity of data available on the rapidly emerging resistance in gonorrhoea, this study was undertaken to look for the resistance pattern in Neisseria gonorrhoea among the clinically suspected cases of gonorrhoea over a period of one year in an STD Referral Centre.

\section{Materials and Methods}

This study included 3213 patients who attended the DVL Out Patient Department and STD Referral centre from June 2017 to May 2018. Ethics committee approval was taken for the study.

\section{Clinical history and examination}

A detailed history was taken regarding the onset of symptoms, presence of discharge or associated symptoms. History of exposure to an infected partner was obtained. Any known history of other Sexually Transmitted Diseases in them and their partners was noted. On examination, in males mucopurulent discharge was present at the urethral meatus. In females with complaints of discharge, an internal and external examination was done.

\section{Sample processing}

The endocervical and urethral discharge samples were collected using sterile dacron swabs. Gram's stain was performed to observe intracellular and extracellular gramnegative diplococci. Culture was performed on blood agar and chocolate agar and placed in a candle jar (5\% CO2) and incubated at 37 ${ }^{0} \mathrm{C}$ for 48 hours. The colonies suspected to be
N. gonorrhoeae were identified by Gram's stain and oxidase test. Figure 1

\section{Antimicrobial Susceptibility testing}

All isolates were tested for susceptibility to penicillin (0.5 IU), ciprofloxacin $(1 \mu \mathrm{gm})$, nalidixic acid $(30 \mu \mathrm{gm})$, ceftriaxone $(0.5$ $\mu \mathrm{gm})$, spectinomycin $(100 \mu \mathrm{g})$, tetracycline $(10 \mu \mathrm{g})$, gentamycin $(10 \mu \mathrm{g})$, azithromycin $(15 \mu \mathrm{g})$ using the Calibrated Dichotomous Sensitivity (CDS) test, following the Australian Gonococcal Surveillance Programme (AGSP) guidelines. Cefixime $(5 \mu \mathrm{g})$ was tested by the CLSI guidelines. The Calibrated Dichotomous Sensitivity test was performed with chocolate agar comprising Columbia agar base. Figure 2 The zone size was measured using the annular radius which is the shortest distance from the edge of the disc to the edge of the confluent growth. Figure 3 Chromogenic cephalosporin test was performed to identify PPNG. WHO reference strains $\mathrm{K}$ and $\mathrm{L}$ were used as control strains.

\section{Results and Discussion}

3213 patents attended the STD\& DVL Out Patient Department, 68 cases were suspected for gonorrhoea, of which $17(25 \%)$ were positive for Neisseria gonorrhoeae and eight (12\%) were Penicillinase Producing Neisseria gonorrhoeae. Table 1. However, among the total culture positive cases, PPNG were $47 \%$ $(8 / 17)$.

Purulent discharge was seen in 52(78\%) and $32(47 \%)$ had associated dysuria. None of the women had any such complaints, they had been tested only after their partners were tested positive. No patient had complications of epididymitis or orchitis on examination.

Diagnosis was made based on clinical features, positive Gram's stain and culture reports. 
Out of 17 cases of gonorrhoea, only one tested positive for HIV. Four other HIV positive cases were among the clinically suspected cases of gonorrhoea that did not grow the organism on culture.

Ciprofloxacin (100\%) and nalidixic acid $(100 \%)$ showed high resistance, followed by penicillin $\mathrm{G}(47 \%)$ and tetracycline $(20 \%)$. All the isolates were susceptible to ceftriaxone (100\%), cefixime (100\%), azithromycin (100\%), gentamycin (100\%) and spectinomycin (100\%). Table 2.

Patients that presented with complaints of urethral or vaginal discharge and dysuria were included in the study. The prevalence of gonorrhoea was found to be $0.52 \%$. Table 3 This is similar to S K Singh et al., and S Muthuswamy et al., with a prevalence of $0.85 \%$ and $2.3 \%$ respectively. (4) ${ }^{(5)}$ However, Lindan et al., showed a prevalence of $5.4 \% .{ }^{(6)}$ 68 patients were suspected of gonorrhoea out of the 3213 patients attending the STD \& DVL OPD, of which 17(25\%) were positive for Neisseria gonorrhoeae (Table 1). However, in S K Singh's study at Gorakhpur from 690 cases only 8 cases were gonorrhoea. (4)

Table.1 PPNG isolated among suspected cases of gonorrhoea

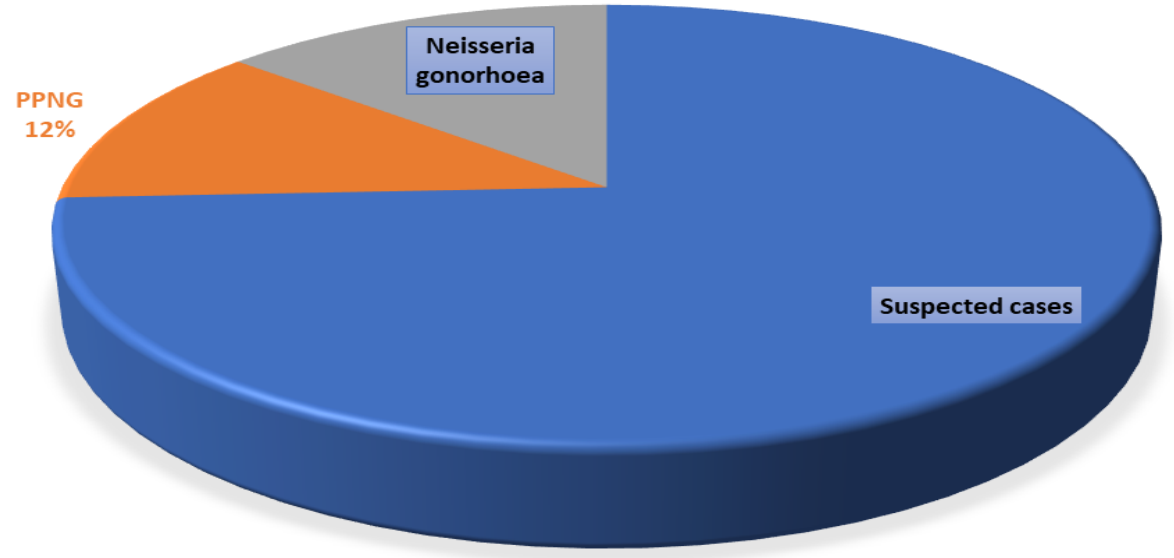

Table.1 Antibiotic susceptibility testing pattern among gonorrhoea isolates

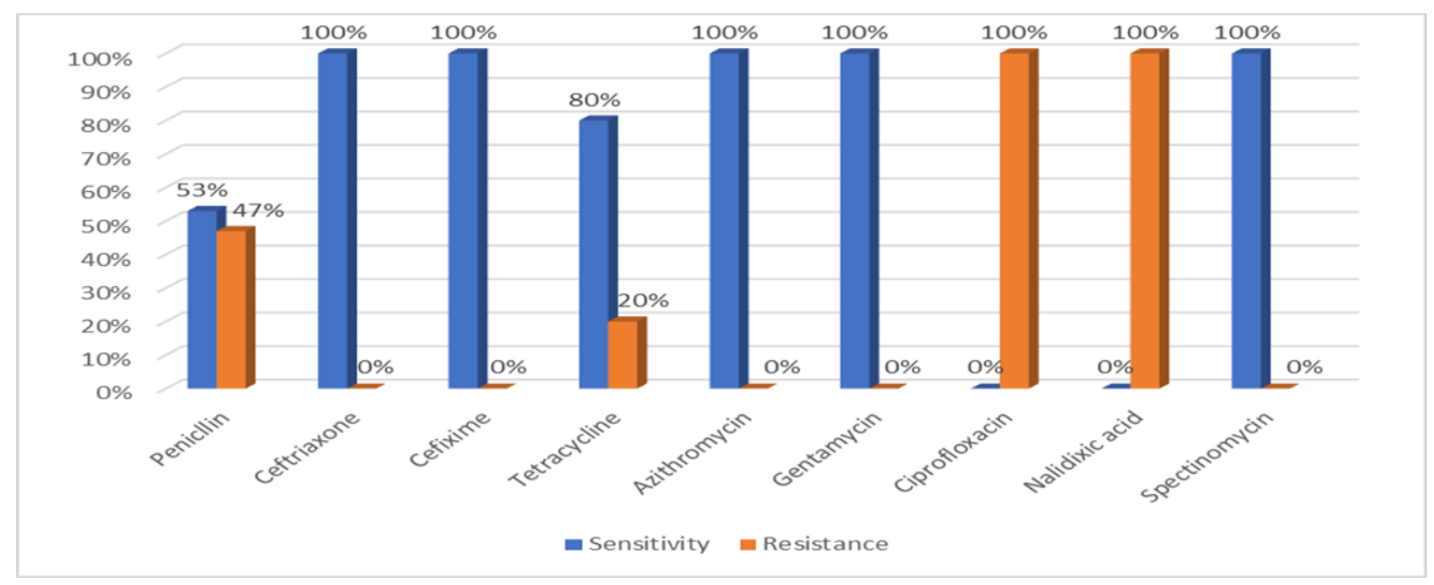


Table.3 Prevalence of gonorrhoea

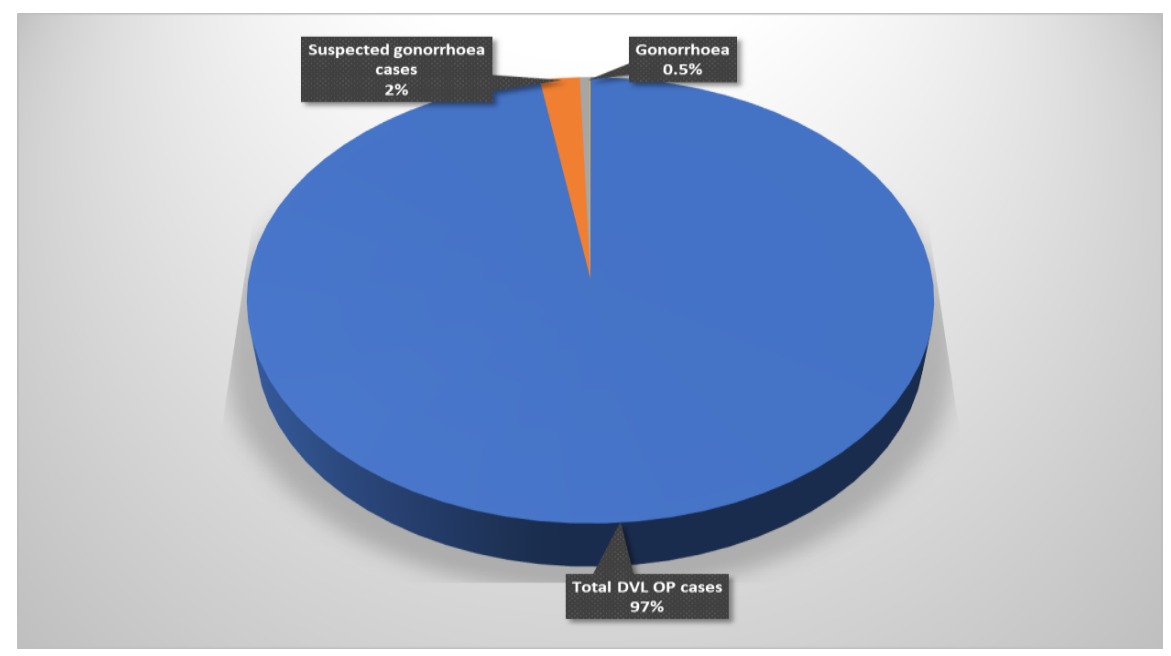

Figure.2 Chocolate agar with colonies of Neisseria gonorrhoeae

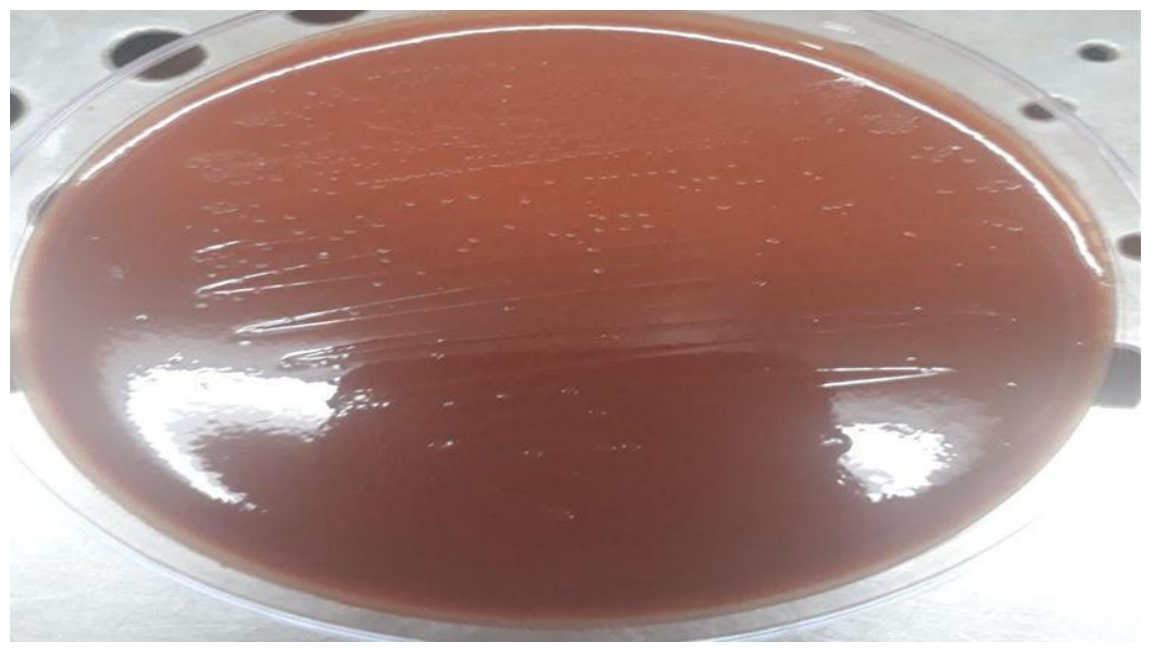

Figure.3 Antimicrobial susceptibility testing of Neisseria gonorrhoeae

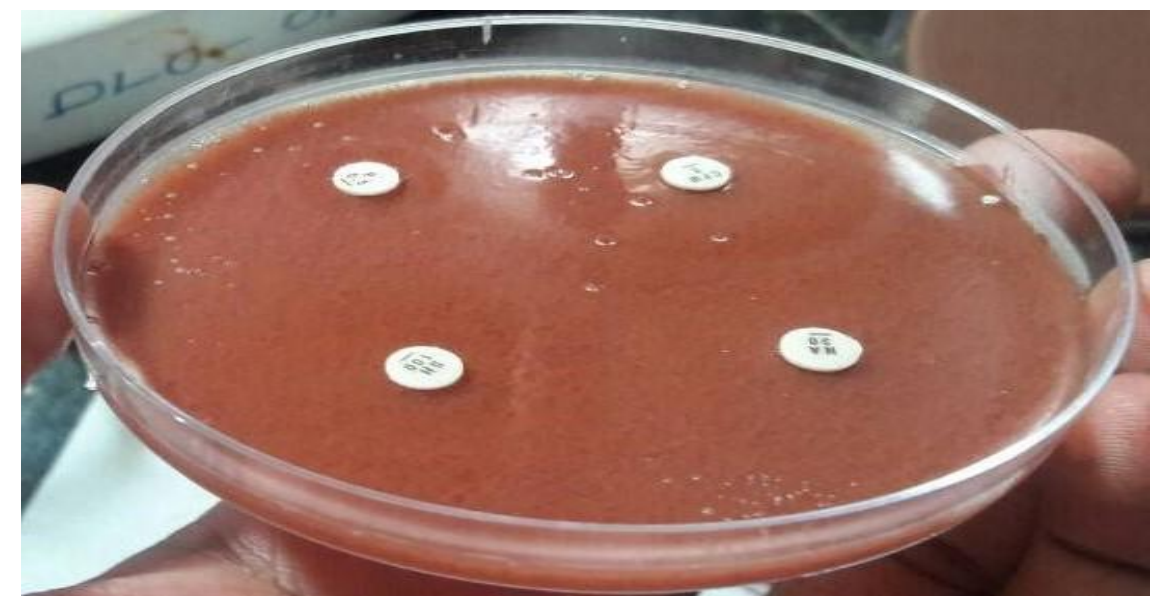


Figure.4 Annular radius measured in CDS method

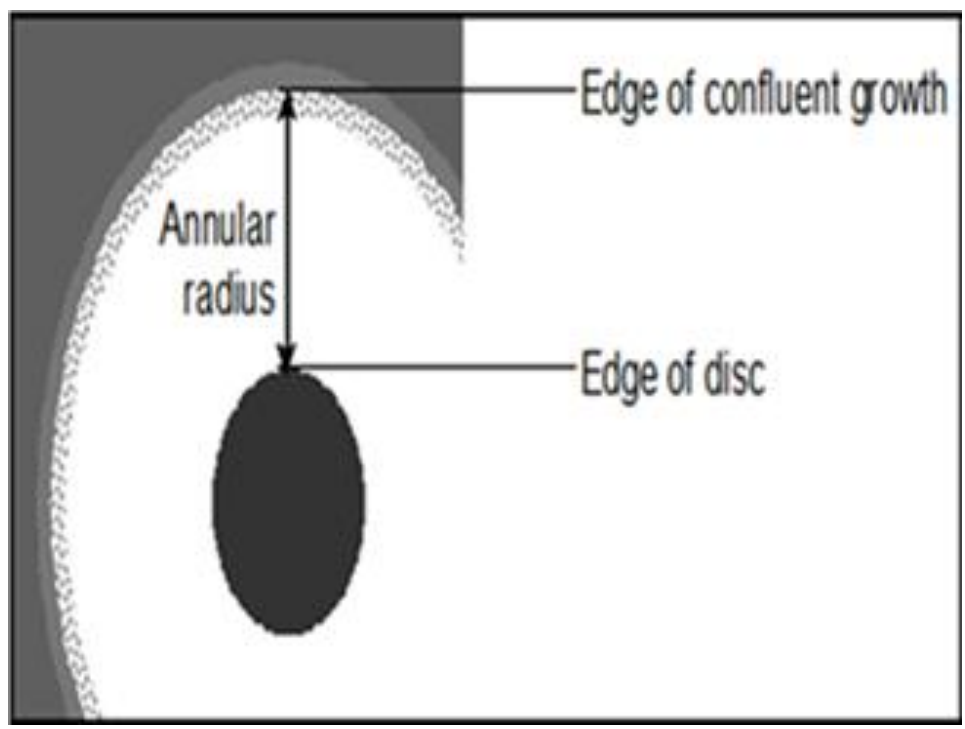

\section{Clinical findings}

All the male patients approached the clinic with complaints of discharge. On examination, 52 had purulent discharge while the rest had serous discharge. This could be due to combined chlamydial and gonococcal infection making it more difficult to diagnose.

The women on the other hand had no such complaints. This is most likely because about $80 \%$ women with gonorrhoea are asymptomatic.

\section{Microbiological findings}

Both Gram's stain and culture were positive for gonorrhea with equal sensitivity. This correlates with Swapna Muthuswamy et al. ${ }^{(5)}$ According to Lindan et al., culture showed a sensitivity of only $62 \%$. The lower performance of culture is probably due to difficulties in maintaining an optimum culture system and preparation of quality media. ${ }^{(6)}$

Among the gonorrhoea cases only one (1/17) was positive for HIV. Rate of HIV co infect on was found to be $17 \%$. This correlates with Bala $\mathrm{M}$ et al., with a rate of $9 \%$. ${ }^{(7)}$ This highlights a relative increase in HIV co infection among the sexually transmitted infections like gonorrhoea.

\section{Antimicrobial susceptibility testing}

By the CDS method of Antibiotic susceptibility testing, penicillin is considered resistant if the annular radius is $\leq 3 \mathrm{~mm}$. Thus, eight isolates were found resistant to penicillin. Furthermore, a colour change from yellow to red when isolated colonies of the isolate were spread on the nitrocefin disc was seen, indicating beta-lactamase production. This defines a gonococcus as Penicillinase Producing Neisseria gonorrhoeae (PPNG). Thus, eight isolates were PPNG in our study. WHO reference strains $\mathrm{K}$ and $\mathrm{L}$ were used as control strains.

In our study, penicillin had $47 \%$ resistance similar to the Sethi S's study of 2017 with a penicillin resistance of $32 \% .{ }^{(8)}$ Table 2 Where as in Bhalla P's study of 1998 , penicillin showed only $8 \%$ resistance, ${ }^{(9)}$ similar to Mishra D's study of 1989. ${ }^{(10)}$ In Bharara T's study, PPNG increased from $8 \%$ in 1995-96 to $20 \%$ in $2004-05$ and $88 \%$ in $2011-2013$. ${ }^{(2)}$ 
This shows that the resistance to penicillin has rapidly increased over the years.

PPNG were found to be $47 \%$ of the total gonorrhoea cases which is similar to Sethi S's study and Bharara T's study that showed 52\% PPNG in 2007-2011 and 88\% PPNG in 20112013 respectively. ${ }^{(2)}{ }^{(8)}$ In contrast, Bhalla et al., in 1998 and Mishra et al., in 1989 showed only 8\% PPNG strains and 52 PPNG cases reported nationwide respectively. ${ }^{(9)(10)}$

This illustrates a rapid increase in PPNG strains in India over the past two decades.

In Bhalla P's study, $28 \%$ of the isolates were TRNG, ${ }^{(9)}$ similar to our study where $20 \%$ of the isolates were TRNG. Penicillin resistance has been showing an increasing trend, however resistance to tetracycline has not shown much change.

Due to high levels of resistance, the fluoroquinolones were abandoned from the CDC-recommended treatment regimens for gonorrhoea in 2017. Currently, High level of resistance to fluoroquinolones was found worldwide. ${ }^{(1)}$ This is in concordance with our study where all the gonococcal isolates were highly resistant to ciprofloxacin $(100 \%)$ and nalidixic acid (100\%) as well as Sethi S's study $(94 \%)$ and Bharara T's study $(84 \%)$ respectively. ${ }^{(2)(8)}$

Azithromycin on the other hand showed $100 \%$ susceptibility. This correlated with Bharara T's study (100\%). ${ }^{(2)}$ Teodora Wi's study illustrates $>5 \%$ decreased susceptibility to ceftriaxone in India in 2014, reporting $\geq 5 \%$ which correlates with Bharara T's study of 2015. ${ }^{(11)(2)}$ However, in our study ceftriaxone and cefixime had $100 \%$ sensitivity, similar to S. Sethi's study. (8) This highlights that azithromycin and ceftriaxone are the current antimicrobial agents appropriate for the treatment of gonorrhoea.

\section{Limitations}

There are some potential limitations to the study. Majority of samples were urethral specimens from male patients (66/68), and only two endocervical swabs from female patients were collected (2/68). Our sample over represents men because men are usually symptomatic and easy to identify for specimen collection. Another concern was that some patients were already on antibiotics before arriving to the clinic.

In conclusion, Neisseria gonorrhoeae is evolving into a superbug with resistance to previously and currently recommended antimicrobials for the treatment of gonorrhoea, which is a major public health concern globally. ${ }^{(1)}$ Neisseria gonorrhoeae exhibited a high level of resistance to penicillin, ciprofloxacin and nalidixic acid. However, tetracycline showed intermediate level of susceptibility. Cefixime, ceftriaxone, azithromycin, gentamycin and spectinomycin which showed highest level of susceptibility are the currently recommended antimicrobials for the treatment of gonorrhoea. The results of our study demonstrated a sharp increase in the percentage of PPNG in India over the past two decades. This calls for a desperate and immediate action to detect and control such increasing resistance. Hence, studying the resistance patterns of gonorrhoea and identification of PPNG strains will greatly help with prescribing the most appropriate antimicrobials for the effective treatment of gonorrhoea and control of its complications.

\section{References}

1. Unemo M, Shafer WM. Antimicrobial Resistance in Neisseria gonorrhoeae in the 21st Century: Past, Evolution, and Future. Clinical Microbiology Reviews 2014; 3: 587-613.

2. Bharara T, Bhalla P, Rawat D, Garg VK, Sardana $\mathrm{K}$, et al., Rising trend of 
antimicrobial resistance among Neisseria gonorrhoeae isolates and the emergence of $\mathrm{N}$. gonorrhoeae isolates with the decreased susceptibility to ceftriaxone. Indian Journal of Medical Microbiology 2015; 33: 39-42.

3. Zheng $\mathrm{H}, \mathrm{Wu} \mathrm{X}$, Huang $\mathrm{J}$, et al., The prevalence and epidemiology of plasmid-mediated penicillin and tetracycline resistance among Neisseria gonorrhoeae isolates in Guangzhou, China, 2002-2012. BMC Infect Dis. 2015; 15 : 412.

4. Singh SK, Kumar N, Gupta AK et al., An epidemiological study of sexually transmitted diseases cases at STD clinic, Gorakhpur. International Journal of Research in Dermatology 2018; 4: 185189.

5. Muthusamy S, Elangovan S. A study on prevalence and antibiotic sensitivity testing methods for Neisseria gonorrhoeae isolates among female outpatients of sexually transmitted infection clinic. Int $J$ Health Allied Sci 2017; 6: 11-4.

6. Lindan C, Mathur M, Kumta S, et al., Utility of pooled urine specimens for detection of Chlamydia trachomatis and Neisseria gonorrhoeae in men attending public sexually transmitted infection clinics in Mumbai, India, by PCR. $J$ Clin Microbiol 2005; 43(4): 1674-7.

7. Bala M, Mullick JB, Muralidhar S, Kumar J, Ramesh V. Gonorrhoea \& its coinfection with other ulcerative, nonulcerative sexually transmitted \& HIV infection in a Regional STD Centre. Indian J Med Res. 2011; 133(3): 346-9.

8. Sethi S, Golparian D, Bala M, et al., Antimicrobial susceptibility and genetic characteristics of Neisseria gonorrhoeae isolates from India, Pakistan and Bhutan in 2007-2011. BMC Infectious Diseases 2013; 10: 1335.

9. Bhalla P, Sethi K, Reddy B S N, Mathur M $\mathrm{D}$ et al., Antimicrobial susceptibility and plasmid profile of Neisseria gonorrhoeae in India (New Delhi). Sexually Transmitted Infections. 1998; 74: 210-212.

10. Mishra D, Singh G. Penicillinaseproducing Neisseria gonorrhoeae in India. Indian J Sex Transm Dis. 1989; 2: 45-7.

11. Wi T, Lahra MM, Ndowa F, et al., Antimicrobial resistance in Neisseria gonorrhoeae: Global surveillance and a call for international collaborative

\section{How to cite this article:}

Syeda Summaiyah and Jyothi Lakshmi, G. 2020. Emerging Resistance in Neisseria gonorrhoeae at an STD Referral Centre. Int.J.Curr.Microbiol.App.Sci. 9(03): 543-549. doi: https://doi.org/10.20546/ijcmas.2020.903.063 